\title{
AVALIAÇÃO DAS PUBLICAÇÕES DE CIRURGIÕES DE COLUNA BRASILEIROS NA ÚLTIMA DÉCADA
}

\author{
EVALUATION OF PUBLICATIONS BY BRAZILIAN SPINE SURGEONS IN THE LAST DECADE \\ CONTRIBUCIÓN CIENTÍFICA DE CIRUJANOS DE COLUMNA BRASILEÑOS ENTRE \\ ENERO DE 2000 Y DICIEMBRE DE 2011
}

Asdrubal Falavigna ${ }^{1}$, Ricardo Vieira Botelho², Alisson Roberto Teles ${ }^{3}$, Pedro Guarise da Silva ${ }^{4}$, Helton Luiz Aparecido Defino ${ }^{5}$

\begin{abstract}
RESUMO
Objetivo: Avaliar a contribuição cientifica de cirurgiões da coluna brasileiros não somente em número de publicações, mas também em relação à qualidade entre Janeiro de 2000 e Dezembro de 2011. Métodos: Uma pesquisa das publicações de cirurgiões da coluna brasileiros em relação à coluna ou medula espinhal foi realizada utilizando uma base de dados online; Pubmed.gov. Os resultados foram limitados para artigos publicados entre Janeiro de 2000 e Dezembro de 2011. Um total de 1.778 artigos foram encontrados após a pesquisa no Medline. Após critérios de exclusão, 206 foram selecionados. A qualidade dos periódicos foi medida através do Fator de Impacto (FI), e do Qualis CAPES e a qualidade do artigo pela classificação de Oxford. Resultados: Um aumento no número de publicações de cirurgiões da coluna brasileiros foi observado. $45,1 \%$ das publicações foram nos últimos 4 anos (2008 - 2011). Estudos clínicos e relatos de caso foram os tipos de artigos mais frequentemente publicados (37,5\% vs 31,1\%). Um aumento no número de publicações brasileiras em periódicos não-brasileiros foi observado nos últimos anos (linear-by-linear association: 5.449, $\mathrm{P}=0.020$ ). A publicação Arquivos de Neuro-psiquiatria foi o periódico em que os artigos foram mais frequentemente publicados ( $n=67,32 \%)$. O Fl das publicações variou de 0.021 a 8.017. A análise da qualidade das publicações demonstrou que a maioria delas forneceu NE 4 ( $N=113,54.9 \%)$ ou $5(N=45,21,8 \%)$. Conclusão: Houve um aumento no número e na qualidade das publicações de cirurgiões da coluna brasileiros nos últimos anos. Além disso, o número de publicações de brasileiros em periódicos não-brasileiros aumentou nos últimos anos.
\end{abstract}

Descritores: Coluna vertebral/cirurgia; Publicações científicas e técnicas; Publicações periódicas/normas; Bibliometria; Pesquisa; Brasil.

\begin{abstract}
Objective: To evaluate the scientific contribution of Brazilian spine surgeons not only in number of publications, but also their quality between January 2000 to December 2011. Methods: A research of publications by Brazilian spinal surgeons on topics concerning the spine or spinal cord was performed using an online data-base; Pubmed.gov. The results were limited to articles published from January 2000 to December 2011. A total of 1,778 articles were identified after a Medline search. After exclusion criteria, 206 papers were selected. The quality of the Journals was assessed with Impact Factor (IF), and the Qualis CAPES, and the article quality using the Oxford classification. Results: An increasing number of publications by Brazilian spine surgeons was observed. 45.1\% of those papers were published during the last 4 years (2008 - 2011). Clinical studies and case reports were the most frequent types of article published (37.5\% vs 31.1\%). An increasing number of Brazilian publications in non-Brazilian journals has been observed in recent years (linear-by-linear association: $5.449, P=0.020$ ). The Arquivos de Neuro-Psiquiatria was the most frequent journal in which the papers were published ( $n=67$, $32 \%)$. The IF of the publications varied from 0.021 to 8.017 . The analysis of quality of the articles using the Oxford classification demonstrated that most of them provided LOE $4(N=113,54.9 \%)$ or $5(N=45,21,8 \%)$. Conclusions: There has been an increasing in the number and quality of publications by Brazilian spine surgeons in recent years. Also, the number of publications by Brazilians in non-Brazilian journals has increased in recent years.
\end{abstract}

Keywords: Spine/surgery; Scientific and technical publications; Periodicals/standards; Bibliometrics; Research; Brazil.

\section{RESUMEN}

Objetivo: Evaluar la contribución científica de cirujanos de columna brasileños, no solamente en número de publicaciones, sino en relación a la calidad, entre enero de 2000 y diciembre de 2011. Métodos: Se realizó una investigación de las publicaciones de cirujanos de columna brasileños en relación a la columna o médula espinal, utilizando una base de datos online: Pubmed.gov. Las búsquedas se limitaron a artículos publicados entre enero de 2000 y diciembre de 2011. Se encontraron 1.778 artículos en la investigación en el Medline. Luego de criterios de exclusión, se seleccionaron 206. La calidad de los periódicos se midió a través del Factor de Impacto (FI) y del Qualis CAPES, y la calidad del artículo, por la clasificación de Oxford. Resultados: Se obsenó un aumento en el número de publicaciones de cirujanos de columna brasileños, siendo que el 45,1\% de las publicaciones se hicieron en los últimos 4 años (2008 - 2011). Estudios clínicos y relatos de caso fueron el tipo de artículos publicados con más frecuencia (37,5\% vs 31,1\%). Hubo aumento en el número de publicaciones brasileñas en periódicos no brasileños en los últimos años (linear-by-linear association: 5,449, $P=0,020)$. Arquivos de Neuro-Psiquiatria fue o periódico en que los artículos se publicaron con más frecuencia ( $n=67,32 \%)$. El factor de impacto de las publicaciones tuvo una variación de 0,021 a 8,017. El análisis de la calidad de las publicaciones demostró que la mayoría de ellas alcanzó nivel de evidencia $4(N=113,54,9 \%)$ o $5(N=45,21,8 \%)$. Conclusiones: Hubo un aumento en el número y en la calidad de las publicaciones de cirujanos de columna brasileños en los últimos años. Además, el número de publicaciones de brasileños ha aumentado a lo largo de los últimos años.

Descriptores: Columna vertebral/cirugía; Publicaciones científicas y técnicas; Publicaciones periódicas/; Investigación/normas; Bibliometría; Brasil.

1. Professor de Neurocirurgia, Coordenador do Curso de Medicina da Universidade de Caxias do Sul - Caxias do Sul, RS, Brasil.

2. Neurocirurgião; Coordenador do Grupo de Cirurgia da Coluna do Serviço de Neurocirurgia do Hospital do Servidor Público Estadual Francisco Morato de Oliveira - São Paulo, SP, Brasil

3. Residente de Neurocirurgia do Hospital São José, Santa Casa de Porto Alegre - Porto Alegre, RS, Brasil.

4. Acadêmico de Medicina, Universidade de Caxias do Sul - Caxias do Sul, RS, Brasil.

5. Professor de Ortopedia eTraumatologia, Faculdade de Medicina de Ribeirão Preto, Universidade de São Paulo - Ribeirão Preto, SP, Brasil.

Trabalho realizado no Serviço de Neurocirurgia da Universidade de Caxias do Sul, RS, Brasil.

Correspondência: Rua General Arcy da Rocha Nóbrega, 401/602 Caxias do Sul, RS, Brasil. 95040-290. asdrubalmd@gmail.com

Recebido em 27/09/2012, aceito em 08/11/2012. 


\section{INTRODUÇÃO}

O principal objetivo da publicação de artigos científicos é dividir informação, sendo ainda mais importante quando as publicações vêm de diferentes continentes, uma vez que isso demonstra que essa descoberta pode ser aplicada por médicos em todo o mundo para resolver suas questões diárias. A educação continuada deve viabilizar aos profissionais acesso as novas informações e mudanças ocorridas em suas áreas, permitindo uma melhora da prática.

Os países de alta renda apresentaram 90,4\% das publicações científicas durante o período de 1992 a 2001, enquanto que 7,9\% foram publicados por países de renda média, incluindo o Brasil com $0,7 \%$, e $2,7 \%$ por países de baixa renda ${ }^{1,2}$. Este acentuado desequilíbrio pode ter várias explicações, tais como, a produção científica em países de maior renda é maior, se comparado com as de menor e média renda, devido ao maior investimento na pesquisa, número elevado de laboratórios e pesquisadores e maior número de atrativos para pesquisadores privilegiados e capacitados que residem em países de menor renda ${ }^{3}$.

A produção científica brasileira, avaliada pelo número de publicações científicas indexadas no Institute for Scientific Information (ISI), vem apresentando um consistente incremento nos últimos anos $^{4}$. A crescente presença do Brasil neste ranking mundial de indexação, da 22a posição em 1998 para $13^{\mathrm{a}}$ em 2008, responde por aproximadamente $2 \%$ do total da produção científica mundial ${ }^{5}$. Heldwein et al. ${ }^{6}$ demonstraram um aumento nas publicações de autores brasileiros em cirurgia geral, com o número de publicações brasileiras subindo de $34^{a}$ posição $(0,07 \%)$ nos anos setenta para a $13^{a}$ posição nos anos $2000(1,47 \%)$. O mesmo foi observado por Tess et al. ${ }^{7}$ com o número de publicações brasileiras em cardiologia aumentando de 77 em 2000 para 109 em 2003. Em 2008, o número de artigos científicos indexados publicados por pesquisadores brasileiros foi de $30.451^{4}$. Dentre as áreas do conhecimento com maior produção científica no Brasil está a Medicina, que responde por aproximadamente $25 \%$ das publicações brasileiras, indexadas no ISI no período entre 1998-2002, seguida pela Física com 15\%, e Química com aproximadamente 10\% .

O objetivo do presente estudo foi avaliar a contribuição científica de cirurgiões da coluna brasileiros não somente em número total de publicações, mas também em relação à qualidade do periódico e do artigo entre Janeiro de 2000 e Dezembro de 2011.

\section{MATERIAS E MÉTODOS}

Realizou-se uma busca sistemática na base de dados Medline de artigos publicados entre Janeiro de 2000 e Dezembro de 2011 que envolviam cirurgiões de coluna brasileiros. Os termos utilizados foram Brazil* AND ("spine" OR "spinal diseases" OR "spinal cord" OR "spinal cord diseases" OR "vertebroplasty" OR "arthrodesis" OR "diskectomy" OR "foraminotomy" OR "laminectomy" OR "denervation" OR "back injuries")

Baseado no título e no resumo foram excluídos artigos não relacionados a cirurgia da coluna, cartas ao editor, artigos sem cirurgiões da coluna, artigos publicados em periódicos brasileiros mas sem autores brasileiros, relatos de caso de pacientes brasileiros sem autores brasileiros e artigos em que não foi possível definir a presença de um cirurgião da coluna (Figure 1). Os artigos selecionados foram avaliados por dois autores, sendo as divergências nas classificações resolvidas por consenso entre os autores.

As variáveis estudadas em cada artigo foram ano de publicação, estado de origem do autor principal, instituição de origem, participação de autor estrangeiro, periódico, país de origem do periódico, classificação Qualis CAPES do periódico, tipo de publicação e patologia estudada. Foram considerados periódicos nacionais todos aqueles produzidos no Brasil, independente da circulação (nacional ou internacional).

A qualidade do periódico foi avaliada pela classificação Qualis CAPES 2012. A Qualis CAPES é um método brasileiro de classificação utilizado entre outros motivos para medir a qualidade da produção científica, graduando os periódicos em diferentes níveis: A1 (mais alto); B1; B2; B3; B4; B5; C (zero) ${ }^{3}$.
A análise estatística foi realizada com o programa SPSS 20. As variáveis categorias foram apresentadas em número e proporção. Análises bivariadas entre as variáveis foram conduzidas com os testes de qui-quadrado e de associação linear.

\section{RESULTADOS}

Um total de 1.778 artigos foram encontrados após a pesquisa na base da dados Medline. A maioria dos trabalhos foi excluído com base nas informações encontradas no título ou no resumo. Dos 690 artigos potencialmente elegíveis para o estudo, a maioria foi excluído por não ser relacionado à cirurgia de coluna ou por não haver cirurgião de coluna brasileiro entre os autores (Figura 1). Após exclusão, 206 artigos foram analisados no estudo.

Em relação à quantidade de publicações envolvendo cirurgiões de coluna brasileiros, observou-se um aumento progressivo nos últimos anos: 45,1\% dos artigos foram publicados entre 2008 e 2011, 39,3\% entre 2004 e 2007, e somente 15,5\% entre 2000 e 2003.

Dos 206 artigos incluídos no estudo, somente 6 (2,9\%) foram realizados com a participação de autores estrangeiros, $3(1,5 \%)$ da América do Norte; 1 (0,5\%) Alemanha; 1 (0,5\%) Italiano; 1 (0,5\%) Canadense e 1 (0,5\%) Francês.

Das diferentes instituições citadas nos artigos, apenas seis $(6,9 \%)$ tinham programas de pós-graduação em neurocirurgia ou ortopedia. Apesar disto, dos 206 artigos incluídos, 74 (36,8\%) estavam ligados a uma dessas instituições.

As publicações foram produzidas por instituições da região Sudeste do país $(71,4 \%)$, seguida pela região Sul $(18,4 \%)$, Nordeste $(5,3 \%)$ e Centro-Oeste (4,9\%). Todas as instituições de pós-graduação em neurocirurgia e ortopedia incluídas na análise se localizam na região Sudeste do país. Em relação à participação de instituição estrangeira, publicação em periódico internacional ou classificação CAPES, não se observou diferença estatisticamente significativa entre as regiões.

Boa parte dos artigos incluídos foram publicados em periódicos brasileiros ( $\mathrm{N}=82 ; 39,8 \%)$. O periódico Arquivos de Neuropsiquiatria (Arq Neuropsiquiatr) publicou 67 (32\%) dos artigos incluídos no estudo. A Figura 2 demonstra os periódicos com pelo menos três artigos publicados de cirurgiões de coluna brasileiros. Periódicos com menos de três artigos foram categorizados como "outros": Pediatric Neurosurgery (1), Revista do Colégio Brasileiro de Cirurgiões (1), Physiotherapy (1), International Orthopedics (2), Journal of Neurosurgical Science (2), Stereotactic and Functional Neurosurgery (2), Acta Neurochirurgica Supplement (2), Journal of Spinal Disorders and Techniques (1), Surgical Neurology International (1), International Journal of General Medicine (1), BMC Emergency Medicine

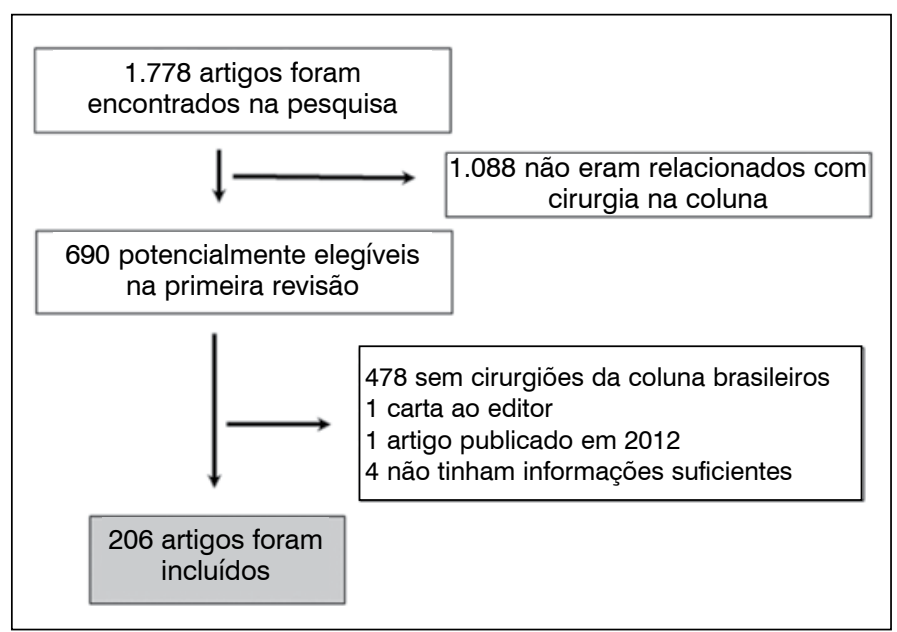

Figura 1. Pesquisa das publicações de cirurgiões da coluna brasileiros em relação a coluna ou medula espinhal utilizando a base de dados online Pubmed.gov. Durante o período de Janeiro de 2000 a Dezembro de 2011 com o total de artigos e critérios de exclusão. 


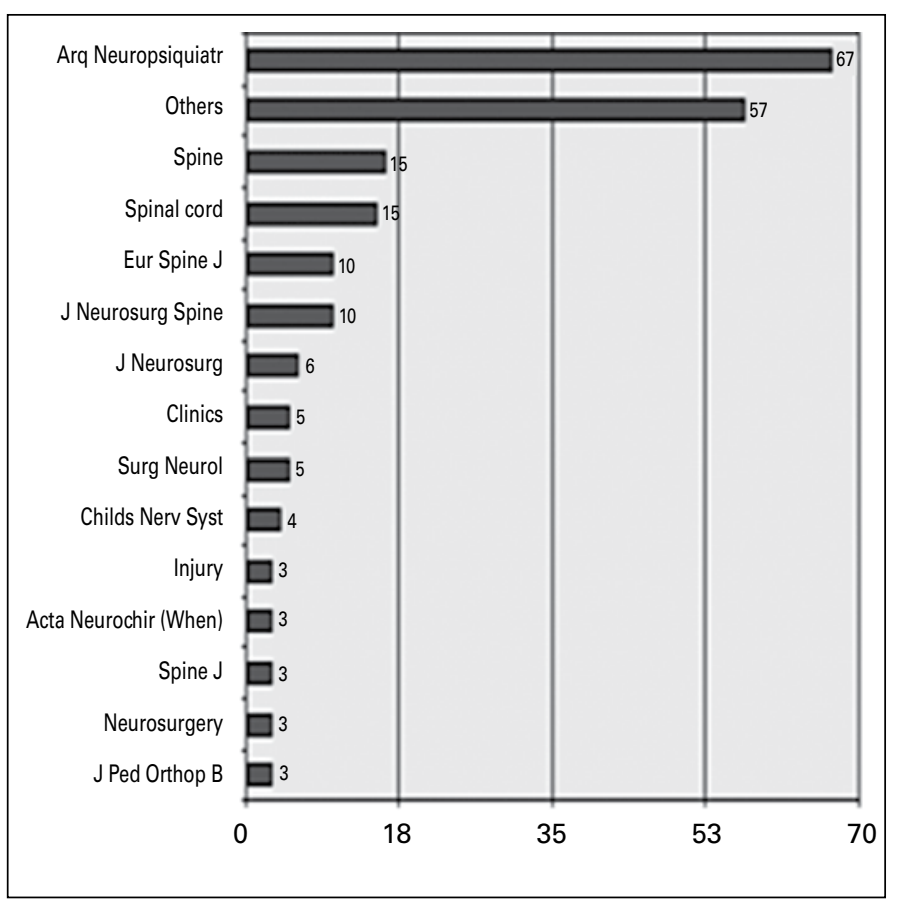

Figura 2. Número de publicações de acordo com o periódico.

(1), Neurosurgical Focus (2), Pediatric Neurology (2), Neurology (1), Revista da Associação Médica Brasileira (1), Journal of Neurosurgery Pediatrics (2), São Paulo Medical Journal (2), Neurology India (1), Journal of Clinical Neuroscience (1), Journal of Trauma (1), Transplantation Procedures (2), Indian Journal of Orthopedics (1), Brazilian Journal of Medical and Biological Research (2), Journal of Craniomaxillofacial Surgery (1), Neurosurgical Review (2), Scandinavian Journal of Infectious Disease (1), Journal of Neuroscience Methods (1), The American Journal of Medical Sciences (1), Tropical Doctor (1), Fetal Diagnosis and Therapy (1), Revista do Hospital de Clínicas da Faculdade de Medicina de São Paulo (1), Neuroscience Letters (1), Artificial Organs (1), British Journal of Sports Medicine (1), Digestive Diseases and Science (1), Revista Brasileira de Anestesiologia (1), International Journal of Neuroscience (1), Revista da Sociedade Brasileira de Medicina Tropical (1), Revista Brasileira de Fisioterapia (1), Journal of Neuroimaging (1), Journal of the American Veterinary Medical Association (1), and Journal of Children 's Orthopaedics (1).

Dos 206 artigos analisados, seis foram publicados em periódicos que não estavam incluídos na classificação Qualis CAPES. Observa-se que a maioria das publicações foram classificadas como B3 e B1. Apenas um artigo foi publicado em periódico com QUALIS A1. Não se observou diferença estatisticamente significativa quando realizadas análises de associação linear entre classificação CAPES e ano de publicação do artigo.

\section{DISCUSSÃO}

Os artigos científicos publicados a cerca de patologias da coluna promoveram melhoras da aplicabilidade prática em relação à ciência básica, diagnóstico e opções de tratamento. Houve um aumento progressivo nos últimos anos da quantidade de publicações envolvendo cirurgiões de coluna brasileiros. A análise quantitativa da produção científica não é o suficiente para determinar a relevâncias da produção científica realizada por cirurgiões da coluna. Porém, o nosso estudo demonstra que além do aumento quantitativo das publicações brasileiras, a qualidade das publicações também aumentou, apesar da maioria das publicações serem classificadas como B3 e B1. Esse aumento da produção científica foi também observado em estudo sobre pesquisadores do CNPq, além da área de Medicina, em outras áreas como Odontologia, Saúde Pública e Fisioterapia ${ }^{9-13}$.
A avaliação do perfil e a produção científica de pesquisadores de Medicina, que têm bolsa de produtividade do Conselho Nacional de Desenvolvimento Científico e Tecnológico (CNPq), de acordo com a área de atuação no triênio de 2006 a 2008 evidenciou que, dos 411 pesquisadores com bolsas ativas, em relação ao curso de graduação, 383 pesquisadores (93,2\%) são formados em Medicina, $11(2,7 \%)$ em Ciências Biológicas, quatro (1\%) em Biomedicina, três $(0,7 \%)$ em Odontologia, três $(0,7 \%)$ em Bioquímica e sete $(1,7 \%)$ em outros $\operatorname{cursos}^{9}$. Existe um equilíbrio entre as diversas áreas de atuação ou especialidade, entretanto existem áreas que apresentam mais de 10 pesquisadores e respondem por aproximadamente 90\% do total de bolsistas da Medicina como: Nefrologia, Cardiologia, Endocrinologia, Neurociências, Infectologia, entre outras ${ }^{9}$.

A participação de autores estrangeiros foi observada somente em 2,9\% das publicações $(n=6 / 206)$, tornando claro a necessidade de um incentivo para estudo multicêntricos internacionais.

Grande parte do aumento do número de publicações no Brasil se deve á expansão no setor de pós-graduação e pesquisa nas últimas décadas. O aumento do número de pós-graduações em Ortopedia e Neurocirurgia nos últimos 10 anos foi um dos responsáveis pelo aumento do número de publicações, uma vez que um dos critérios para a avaliação destas instituições é o número a qualidade dos artigos publicados por ano. Este dado foi observado no presente trabalho, pois dos 206 trabalhos incluídos, 74 (36,8\%) estavam vinculados a uma dessas instituições, sendo que das 87 instituições que foram relacionadas nos artigos apenas $6(6,9 \%)$ tinham programas de pós-graduação em Neurocirurgia e Ortopedia e todas localizadas na região Sudeste do pais.

Quatro Estados da Federação são responsáveis por 90\% dos pesquisadores $\mathrm{CNPq}(\mathrm{SP}, \mathrm{RJ}, \mathrm{RS}, \mathrm{MG})^{9,12}$. A análise da razão de número de bolsistas de produtividade em pesquisa por habitantes mostra que a média nacional é de 2,14 bolsistas em medicina por milhão de habitantes. ${ }^{9}$ Entretanto apenas três Estados apresentam uma razão bolsistas/milhão de habitantes acima da média nacional (São Paulo, Rio Grande do Sul e Rio de Janeiro) ${ }^{9,12}$. Oito instituições são responsáveis por aproximadamente $80 \%$ dos pesquisadores, destacando-se USP $(30,7 \%)$ e UNIFESP $(17 \%)^{9}$. Estes mesmos dados foram observados no nosso estudo, onde as publicações foram produzidas por instituições da região Sudeste do país $(71,4 \%)$, seguida pela região Sul $(18,4 \%)$, Nordeste $(5,3 \%)$ e Centro-Oeste $(4,9 \%)$.

O periódico Arquivos de Neuropsiquiatria foi o periódico com o maior número de publicações de cirurgiões da coluna brasileiros, sendo responsável por $32,5 \%$ de todos os artigos publicados e $39,8 \%$ dos artigos publicados em periódicos nacionais desde os anos 2000. Parece haver uma percepção generalizada entre cientistas brasileiros de que existe preconceito contra trabalhos da América do Sul por parte de editores e revisores de revistas publicadas em países da América do Norte, particularmente nos países de língua inglesa que concentram a maior parte dos periódicos de alto impacto ${ }^{3}$. A língua Inglesa, a presença de uma minoria $(0 \%$ a $7,2 \%)$ dos conselheiros editoriais provenientes da América do Sul nas revistas médicas internacionais de grande impacto ${ }^{14,15}$, e vieses devido a nacionalidade podem ser fatores que influenciam na taxa de aprovação de trabalhos brasileiros em revistas internacionais ${ }^{16,17}$. Apesar disto, não parece ser justo atribuir toda a responsabilidade aos editores uma vez que os problemas mais frequentemente mencionados para publicação internacional incluem deficiências na redação em inglês, a má qualidade geral de alguns artigos e problemas de saúde com baixa relevância internacional ${ }^{3}$

O aumento das publicações em periódicos indexados, tanto nacionais quanto internacionais, indica que os pesquisadores estão se preocupando em relação à visibilidade de suas publicações. Muitos fatores levam a uma promoção da produção científica brasileira: (a) as agências de fomente e comitês institucionais responsáveis por eleger candidatos para posições acadêmicas, normalmente baseiam suas decisões pelo número de publicações científicas em periódicos de prestígio internacional; (b) aumento no aporte de recursos de fomento para pesquisa das agências federais, especialmente nos últimos anos, juntamente com a adesão de financiamen- 
to dos estados; (c) o crescimento do número e do valor das bolsas federais, corrigidos em 2004 e 2008 em 67\% (variação nominal); (d) o crescimento de titulados na pós-graduação, sobretudo no doutorado, onde se dá a maior parte da produção científica brasileira; (e) a cobrança de melhor desempenho individual dos pesquisadores na avaliação por todas as agências de fomento; ( $f$ ) aperfeiçoamento do sistema de avaliação da pós-graduação pela CAPES para medir a produção científica das instituições, a qual prioriza o número e a qualidade dos artigos publicados para conceituar os programas nacionais ${ }^{18,19}$; (g) a criação do Programa Qualis da CAPES, que classifica as revistas estrangeiras e brasileiras para orientar a avaliação da CAPES; (h) maior competição entre pesquisadores com bolsa de produtividade em pesquisa, incentivando tanto a formação de novos pesquisadores como a publicação de artigos em periódicos de impacto ${ }^{9}$; e (i) maior oportunidade de contatos entre os profissionais ${ }^{20}$

Existem medidas específicas que podem aumentar a possibilidade de publicação no exterior, como: (a) investir na qualidade do texto e contratar redatores científicos especializados, cuja língua nativa seja o inglês; (b) argumentar claramente a contribuição do estudo brasileiro para a literatura internacional; (c) seguir rigorosamente as normas de preparação do manuscrito da revista; (d) não ser prolixo e escrever artigos curtos e objetivos; (e) não subestimar a carta de apresentação do artigo, por ser a melhor oportunidade de convencer o editor de que o artigo é apropriado para a revista; e (f) revisar e aprimorar o resumo e a metodologia ${ }^{3}$. Importante é sempre tentar ver sua publicação em revistas de grande visibilidade pois, em última instância, o grande problema de ter trabalhos recusados é o autor ser desconhecido. Entretanto, o fato de que autores brasileiros estão atualmente publicando internacionalmente mostra que estes obstáculos não são intransponíveis. Um reflexo disto são as evidentes mudanças nas atitudes de algumas revistas internacionais e a indicação de consultores editoriais internacionais, incluindo o Brasil ${ }^{2,3}$.

\section{CONCLUSÃO}

Vem havendo um aumento no número e na qualidade das publicações por cirurgiões da coluna brasileiros nos últimos anos. Embora o inglês não seja a língua nativa no Brasil, um aumento no número de publicações em periódicos internacionais também vem sendo observada. Existe uma expectativa que junto com o aumento no número de pós-graduações em Neurocirurgia e Ortopedia, exista uma melhor capacitação para pesquisa, e, consequentemente, maior quantidade e qualidade dos artigos publicados.

\section{REFERÊNCIAS}

1. Paraje G, Sadana R, Karam G. Public health. Increasing international gaps in health-related publications. Science. 2005;308(5724):959-60.

2. Sumathipala A, Siribaddana S, Patel V. Under-representation of developing countries in the research literature: ethical issues arising from a survey of five leading medical journals. BMC Med Ethics. 2004 Oct 4;5:E5.

3. Victora CG, Moreira CB. [North-South relations in scientific publications: editorial racism?]. Rev Saude Publica. 2006;40:36-42.

4. Indicadores nacionais de ciência e tecnologia (C\&T): comparações internacionais produção científica. 2009. Disponível em: http://www.mct.gov.br/index.php/content/ view/5710.html.). (Accessado em: ago. 12, 2012).

5. Brazilian Science on the Rise. Science Watch. 2009. Disponivel em: http://sciencewatch. com/ana/fea/pdf/09julaugFea.pdf.). (Accessado em: ago. 12, 2012).

6. Heldwein FL, Hartmann AA, Kalil AN, Neves BV, Ratti GS, Beber MC Jr, et al. Cited Brazilian papers in general surgery between 1970 and 2009. Clinics (Sao Paulo). 2010 May; 65(5):521-9.

7. Tess BH, Furuie SS, Castro RC, Barreto Mdo C, Nobre MR. Assessing the scientific research productivity of a Brazilian healthcare institution: a case study at the Heart Institute of São Paulo, Brazil. Clinics (Sao Paulo). 2009;64(6):571-6.

8. Nitrini R. The scientific production of Brazilian neurologists: 1995-2004. Arq Neuropsiquiatr. 2006;64(2B):538-42.

9. Martelli-Junior H, Martelli DR, Quirino IG, Oliveira MC, Lima LS, Oliveira EA. CNPq-supported medical researchers: a comparative study of research areas. Rev Assoc Med Bras. 2010;56(4):478-83

10. Barata RB, Goldbaum M. [A profile of researchers in public health with productivity

grants from the Brazilian National Research Council (CNPq)]. Cad Saude Publica. 2003;19(6):1863-76.

11. Cavalcante RA, Barbosa DR, Bonan PRF, Pires MBO, Martelli-Junior H. Perfil dos pesquisadores da área de odontologia no Conselho Nacional de Desenvolvimento Científico e Tecnológico (CNPq). Rev Bras Epidemiol. 2008;11:106-13.

12. Santos NCF, Candido LFO, Kuppens CL. Produtividade em pesquisa do CNPq: análise do perfil dos pesquisadores da química. Quimica Nova. 2010:489-95.

13. Santos SMC, Lima LS, Martelli DRB, Junior HM. Perfil dos pesquisadores da Saúde Coletiva no Conselho Nacional de Desenvolvimento Científico e Tecnológico. Rev Saúde Coletiva. 2009;19.

14. Horton R. Medical journals: evidence of bias against the diseases of poverty. Lancet. 2003;361(9359):712-3

15. Saxena S, Levav I, Maulik P, Saraceno B. How international are the editorial boards of leading psychiatry journals? Lancet. 2003;361(9357):609.

16. Callaham M, Wears RL, Weber E. Journal prestige, publication bias, and other characteristics associated with citation of published studies in peer-reviewed journals. JAMA. 2002;287(21):2847-50

17. Meneghini R, Packer AL, Nassi-Calò L. Articles by latin american authors in prestigious journals have fewer citations. PLoS One. 2008;3(11):e3804.

18. Deheinzelin D, Caramelli B. [ Scientific production, post-graduate education and Revista da Associação Médica Brasileira]. Rev Assoc Med Bras. 2007;53(6):471-2.

19. Qualis Periódicos. 2012. Disponível em: http://www.capes.gov.br/avaliacao/qualis\#menu.) (Accessado em: Apr. 28, 2012)

20. Goldenberg S. Part of the history of surgical research in Brazil: historical evolution of the journal Acta Cirurgica Brasileira (1986-2005). Acta Cir Bras. 2008;23:1-3. 